\title{
Article \\ COVID-19 Pandemic in a Vulnerable Population: Prevalence and Correlates of Anxiety
}

\author{
Reham Shalaby ${ }^{1}{ }^{(}$, Ejemai Eboreime $\left.{ }^{1}{ }^{(}\right)$, Nnamdi Nkire ${ }^{1}$, Belinda Agyapong ${ }^{1,2}{ }^{1}$, Hannah Pazderka ${ }^{1}$, \\ Gloria Obuobi-Donkor ${ }^{1}{ }^{\circledR}$, Medard Kofi Adu ${ }^{1}$, Wanying Mao ${ }^{1}$, Ernest Owusu $\left.{ }^{1}{ }^{(}\right)$, Folajinmi Oluwasina ${ }^{1}$ \\ and Vincent I. O. Agyapong $1,3, * \mathbb{C}$
}

1 Department of Psychiatry, University of Alberta, Edmonton, AB T6G 2B7, Canada; rshalaby@ualberta.ca (R.S.); eboreime@ualberta.ca (E.E.); Nnamdi.Nkire@albertahealthservices.ca (N.N.); bagyapon@ualberta.ca (B.A.); hannah@ualberta.ca (H.P.); obuobido@ualberta.ca (G.O.-D.); medard@ualberta.ca (M.K.A.); wmao2@ualberta.ca (W.M.); eowusu2@ualberta.ca (E.O.); folajinm@ualberta.ca (F.O.)

2 Global Psychological E-Health Foundation, Edmonton, AB T6G 2B7, Canada

3 Department of Psychiatry, Faculty of Medicine, Dalhousie University, Halifax, NS B3H 2E2, Canada

* Correspondence: agyapong@ualberta.ca or vn602367@dal.ca; Tel.: +1-780-215-7771; Fax: +1-902-473-4887

\section{check for}

updates

Citation: Shalaby, R.; Eboreime, E.; Nkire, N.; Agyapong, B.; Pazderka, H.; Obuobi-Donkor, G.; Adu, M.K.; Mao, W.; Owusu, E.; Oluwasina, F.; et al. COVID-19 Pandemic in a

Vulnerable Population: Prevalence and Correlates of Anxiety. Behav. Sci. 2022, 12, 13. https://doi.org/

$10.3390 /$ bs12010013

Academic Editor: Valentina

Echeverria

Received: 23 November 2021

Accepted: 9 January 2022

Published: 13 January 2022

Publisher's Note: MDPI stays neutral with regard to jurisdictional claims in published maps and institutional affiliations.

Copyright: (C) 2022 by the authors. Licensee MDPI, Basel, Switzerland. This article is an open access article distributed under the terms and conditions of the Creative Commons Attribution (CC BY) license (https:// creativecommons.org/licenses/by/ $4.0 /)$.

\begin{abstract}
Background: The COVID-19 pandemic has produced negative mental health outcomes. These effects were more prominent in vulnerable communities that experienced prior similar disasters. The study aimed to examine the likelihood and correlates of anxiety symptoms among Fort McMurray (FMM) residents, during the COVID-19 pandemic. Methods: A cross-sectional online survey questionnaire was applied between 24 April and 2 June 2021, at FMM, to gather sociodemographic, COVID-19, and clinical information, including generalized anxiety disorder (using GAD-7 scale). Results: Overall, 186 individuals completed the survey (response rate 74.7\%). Most of the respondents were females $(159,85.5 \%)$; above 40 years $(98,52.7 \%)$; employed $(175,94.1 \%)$; and in relationship $(132,71 \%)$. The prevalence of moderate-to-severe anxiety was $(42.5 \%, 71)$ on GAD-7 self-reported scale. Subscribers who reported that they would like to receive mental health support; have received no family support since COVID-19 declaration; and have lost their job during the pandemic were all more likely to report moderate-to-severe anxiety ( $\mathrm{OR}=3.39 ; 95 \% \mathrm{CI}: 1.29-8.88),(\mathrm{OR}=4.85$; 95\% CI: 1.56-15.03), and (OR $=4.40 ; 95 \%$ CI: 1.01-19.24), respectively. Conclusions: Anxiety levels were high among FMM residents, compared to levels before COVID-19. Clinical and social factors significantly predicted likely anxiety in the Fort McMurray population, during the COVID-19 pandemic. It is imperative that resources are mobilized to support vulnerable communities during the COVID-19 pandemic.
\end{abstract}

Keywords: anxiety; trauma; COVID-19; cross-sectional; online survey; Fort McMurray

\section{Introduction}

Uncertainty and fear as consequences of SARS-CoV-2 infection have increased, producing profound effects on the individual well-being and mental health worldwide. Higher levels of depression, anxiety, and stress have been observed in community members, including healthcare workers [1,2] students [3,4], seniors [5], and patients with health problems including mental health conditions [3], and COVID-19 infection [5]. According to a recent systematic review and meta-analysis by Salari et al., COVID-19 has threatened the mental health of nearly one-third of the general population [6]. The pooled prevalence of stress, depression, and anxiety was estimated at $29.6 \%, 33.7 \%$, and $31.9 \%$, respectively, among the general population during the pandemic [6]. These figures were essentially higher among COVID-19 patients, in two recent systematic reviews and meta-analyses by Pashazadeh Kan. et al. and Deng. et al. [5,7]. While natural disasters could, and do, affect different populations, a subset of exposed people usually incur clinically significant mental health symptoms of stress, depression, post-traumatic stress disorder (PTSD), and 
anxiety $[8,9]$. The available data indicate that people who closely followed COVID-19 news became more anxious and distressed, particularly when the information was exaggerated or fabricated $[10,11]$.

Emerging evidence from studies on the COVID-19 pandemic or similar disasters, suggests that several factors are implicated in the development of mental health and anxiety symptoms; for example, sociodemographic characteristics, such as age, gender, or receiving inadequate social support have significantly impacted mental health outcomes during such difficult times [5,12-14]. Additionally, having an infected family member with COVID-19 or excessive fear of becoming infected were also associated with stressrelated symptoms [14-16]. Furthermore, having an existing mental health condition such as depression or anxiety may present an additional risk, which may contribute to potential exacerbation of the symptoms or relapsing due to service disruption or the need for isolation [13-17]. The received support during the current pandemic was also a significant protector against mental health symptoms. In a previous research [14], authors reported that people who did not receive family support or limited support were more than five times as likely to develop symptoms of post-traumatic stress disorder, compared to those who received absolute support.

The region of Fort McMurray (FMM) is situated in the north of the province of Alberta in Western Canada. By May 2021, the region of FMM had experienced three major disasters over five consecutive years. These include the wildfires associated with an evacuation order in 2016, and flooding and COVID-19 in 2020. Such traumatic experiences are believed to have adversely impacted the mental health and general wellbeing of survivors $[9,18]$. The one-month prevalence of anxiety symptoms [19], PTSD [20], and depressive symptoms [21] were estimated at $19.8 \%, 12.8 \%$, and $14.8 \%$, respectively, six months after the wildfire [19]. Compared to a control population, the prevalence of mental health symptoms remained high among adolescents 18 months after the wildfire [18,22]. In 2020, the flooding affected over 1200 structures, costing damages worth $\$ 375$ million [23]. The physical and economic cost of these experiences is often accompanying emotional difficulties and stress [22,23].

Given the aforementioned information, it is generally expected that there will be a rise in mental health problems due to the burden posed by these traumatic experiences. It is unclear, however, how prevalent the mental health symptoms are among FMM population during the COVID-19 pandemic. In this context, the goal of this study was to examine the prevalence and potential correlates of moderate-to-high anxiety during the month of May 2021 when FMM was considered to be a hotspot for the COVID-19 pandemic in Alberta, Canada [24]. Our specific research questions included: (1) what was the prevalence of moderate-to-high anxiety among residents of Fort McMurray during the month of May 2021 when the city was a hot spot for COVID-19 infections? (2) What were the sociodemographic, clinical, and COVID-19-related predictors for moderate-to-high anxiety in residents of Fort McMurray during the peak of the pandemic? We hypothesize that the prevalence of moderate-to-high anxiety symptoms in residents of FMM is higher than the prevalence reported for the general population in Canada and in other jurisdictions during the pandemic. We also hypothesize, that in addition to sociodemographic and clinical characteristics, there would be COVID-19-related variables that are independently associated with moderate-to-high anxiety symptoms in the respondents.

The conceptual framework explaining the anticipated high prevalence of moderateto-high anxiety in Fort McMurray in comparison to those of the general public in Canada during the pandemic was designed by the study authors and based on empirical evidence that the history of frequent disasters or major traumas may increase levels of mental health conditions $[6,9,18,20]$, and therefore, the anxiety level may be increased under these circumstances and exceeding the levels reported in other communities during the same pandemic (Figure 1). Additionally, the framework suggests a number of potential risk factors that may predispose toward high anxiety levels during the COVID-19 pandemic (Figure 1). 


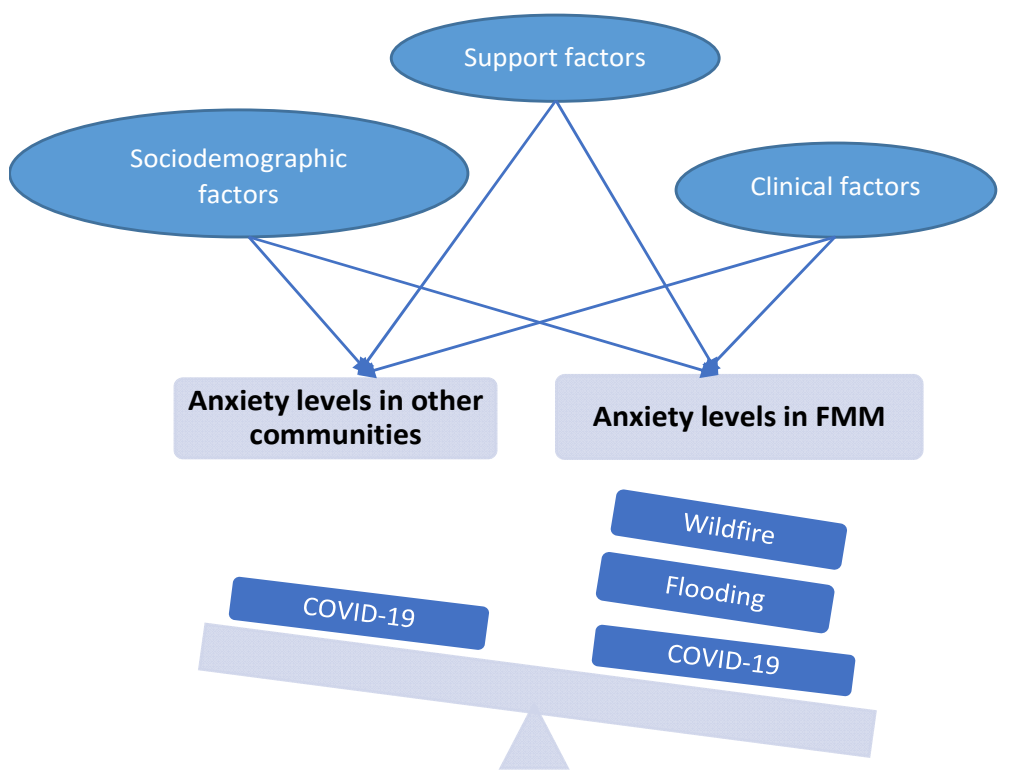

Figure 1. Conceptual framework for the prospective impact of multiple traumas and the predictive factors for high anxiety levels in Fort McMurray (FMM) community versus other communities.

\section{Materials and Methods}

\subsection{Study Setting and Design}

The study data were collected through an online survey completed by people in Fort McMurray (FMM), Canada. FMM represents the urban service area of the Northern Alberta Regional Municipality of Wood Buffalo, with a diverse population of 111,687 as of the 2018 census [25], mainly in temporary project accommodations and in adjoining rural communities [20]. Through a cross-sectional survey introduced from 24 April to 2 June 2021, a self-administered questionnaire was sent to residents of FMM via REDCap [26]. The questionnaire was distributed randomly via email using government, school, occupational, and community platforms. Consent was implied, by completing the survey. Study approval was granted by Alberta Health Research Ethics Committee (Pro00066054).

\subsection{Outcome Measure}

The Generalized Anxiety Disorder-7 (GAD-7) scale was used to assess likely anxiety in the respondents. The scale consists of seven self-reported items that define the symptoms of GAD. Items are rated on a 4-point Likert-type scale $(0=$ not at all to $3=$ nearly every day $)$. Scores range from 0 to 21 with higher scores indicating more severe GAD symptoms [27]. Patients with a GAD-7 score of 10 or more were deemed to have likely anxiety. Two categories were thus generated: Low anxiety $<10$ and moderate-to-high anxiety $\geq 10$. In a heterogeneous clinical population, the GAD-7 has excellent internal consistency and a one-factor structure [27], indicating that its items are all representative of one construct. The GAD-7 is suggested as a valid tool for screening and assessing the severity of GAD in clinical practice and research [28].

\subsection{Statistical Analysis}

Data were analyzed using SPSS Version 25 (IBM Corp 2011) [29]. Descriptive statistics were administered for demographic, clinical, and COVID-19-related variables based on the relationship status. Chi-squared analysis was run to examine all the variables in relation to the likely anxiety categorical variable (low and moderate-to-high anxiety). Logistic regression analysis was employed to identify significant predictors of likely anxiety. The model included the significant $(p<0.05)$ or near significant $(0.1 \geq p \geq 0.05)$ variables with the likely anxiety, obtained from the univariate analysis. Odds ratios (OR) and confidence 
intervals were reported, determining the predictor variables for respondents to self-report likely anxiety during the COVID-19 pandemic, controlling for the rest of the variables.

Correlational analysis was performed to exclude any strong intercorrelations (Spearman's correlation coefficient from 0.7 to 1.0 or from -0.7 to -1.0 ) among predictor variables. No imputation was applied, and reported data represent the complete responses.

\section{Results}

Of the 249 individuals who clicked on the survey link, 186 completed the survey, yielding a response rate of $74.7 \%$. Descriptive information of demographic, clinical, and COVID-19-related data on the full sample of patients $(\mathrm{N}=186)$ were examined against the relationship status of the participants (Table 1). Overall, 71\% (132) reported that they were in a relationship (married, cohabiting, or partnered) while $29 \%$ (54) reported they were not in a relationship (divorced, widowed, or single). From Table 1, most respondents $85.5 \%$ (159) were females; $52.7 \%$ (98) were above 40 years of age; $94.1 \%$ (175) were employed; $78 \%$ (145) owned their own homes; $48.4 \%$ (90) reported having no mental health diagnosis, while $64.5 \%$ (120) reported not receiving psychotropic medications; $38.7 \%$ (72) reported receiving mental health counseling; and $52.7 \%$ (98) reported they would like to receive mental health counseling.

Table 1. Demographic profile, clinical characteristics, and support received by the study population.

\begin{tabular}{|c|c|c|c|}
\hline Variables & $\begin{array}{c}\text { in a } \\
\text { Relationship } \\
\text { n (\%) }\end{array}$ & $\begin{array}{c}\text { Not in a } \\
\text { Relationship } \\
\text { n (\%) }\end{array}$ & $\begin{array}{l}\text { Total } \\
\text { n (\%) }\end{array}$ \\
\hline \multicolumn{4}{|l|}{ Gender } \\
\hline Male & $17(12.9)$ & $10(18.5)$ & $27(14.5)$ \\
\hline Female & $115(87.1)$ & $44(81.5)$ & $159(85.5)$ \\
\hline \multicolumn{4}{|l|}{ Age categories } \\
\hline$\leq 40 \mathrm{y}$ & $62(47.0)$ & $26(48.1)$ & $88(47.3)$ \\
\hline$>40 y$ & $70(53.0)$ & 28 (51.9) & $98(52.7)$ \\
\hline \multicolumn{4}{|l|}{ Employment status } \\
\hline Employed & $125(94.7)$ & $50(92.6)$ & $175(94.1)$ \\
\hline Unemployed & $7(5.3)$ & $4(07.4)$ & $11(5.9)$ \\
\hline \multicolumn{4}{|l|}{ Housing status } \\
\hline Own home & $113(85.6)$ & $32(59.3)$ & $145(78.0)$ \\
\hline Renting & $19(14.4)$ & $22(40.7)$ & $41(22.0)$ \\
\hline \multicolumn{4}{|l|}{$\begin{array}{l}\text { History of mental health } \\
\text { diagnosis from a health } \\
\text { professional }\end{array}$} \\
\hline Depression & $37(28.0)$ & $21(38.9)$ & $58(31.2)$ \\
\hline Bipolar Disorder & $3(2.3)$ & $3(5.6)$ & $6(3.2)$ \\
\hline Anxiety & $54(40.9)$ & $24(44.4)$ & $78(41.9)$ \\
\hline Schizophrenia & $0(0.0)$ & $0(0.0)$ & $0(0.0)$ \\
\hline Personality Disorder & $0(0.0)$ & $2(53.7)$ & $2(1.1)$ \\
\hline Other & $12(9.1)$ & $5(9.3)$ & $17(9.1)$ \\
\hline No mental health diagnosis & $69(52.3)$ & $21(38.9)$ & $90(48.4)$ \\
\hline \multicolumn{4}{|l|}{$\begin{array}{l}\text { History of psychotropic } \\
\text { medications }\end{array}$} \\
\hline Antidepressants & $34(25.8)$ & $25(46.3)$ & $59(31.7)$ \\
\hline Antipsychotics & $2(1.5)$ & $2(3.7)$ & $4(2.2)$ \\
\hline Benzodiazepines & $3(2.3)$ & $1(1.9)$ & $4(2.2)$ \\
\hline Mood stabilizers & $5(3.8)$ & $7(13.0)$ & $12(6.5)$ \\
\hline Sleeping tablets & $12(9.1)$ & $9(16.7)$ & $21(11.3)$ \\
\hline Other & $2(1.5)$ & $1(1.9)$ & $3(1.6)$ \\
\hline On no psychotropic medication & $92(69.7)$ & $28(51.9)$ & $120(64.5)$ \\
\hline
\end{tabular}


Table 1. Cont.

\begin{tabular}{|c|c|c|c|}
\hline Variables & $\begin{array}{c}\text { in a } \\
\text { Relationship } \\
\text { n (\%) }\end{array}$ & $\begin{array}{c}\text { Not in a } \\
\text { Relationship } \\
\text { n (\%) }\end{array}$ & $\begin{array}{l}\text { Total } \\
\text { n (\%) }\end{array}$ \\
\hline $\begin{array}{l}\text { Respondents received MH } \\
\text { counselling in the past year }\end{array}$ & $48(36.4)$ & $24(44.4)$ & $72(38.7)$ \\
\hline $\begin{array}{l}\text { Respondents would like to } \\
\text { receive MH counselling }\end{array}$ & $66(50.0)$ & $32(59.3)$ & $98(52.7)$ \\
\hline $\begin{array}{l}\text { Respondents who have been } \\
\text { fearful about contracting the } \\
\text { coronavirus during the pandemic }\end{array}$ & $113(89.7)$ & $47(97.9)$ & $160(92.0)$ \\
\hline $\begin{array}{l}\text { Respondents who have been } \\
\text { fearful about their close friends or } \\
\text { family members contracting the } \\
\text { coronavirus during the pandemic }\end{array}$ & $121(96.0)$ & 47 (97.9) & $168(96.6)$ \\
\hline $\begin{array}{l}\text { Respondents who reported } \\
\text { having close friends or family } \\
\text { members who have been sick } \\
\text { from the coronavirus disease }\end{array}$ & $94(75.8)$ & $30(62.5)$ & $124(72.1)$ \\
\hline $\begin{array}{l}\text { Have you had to self-isolate or } \\
\text { self-quarantine due to COVID-19 } \\
\text { symptoms, recent travel, or } \\
\text { because you were in contact with } \\
\text { someone who may have } \\
\text { COVID-19? }\end{array}$ & & & \\
\hline 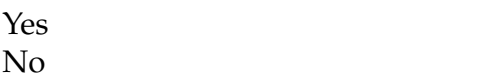 & $\begin{array}{l}77(61.6) \\
48(38.4)\end{array}$ & $\begin{array}{l}27(56.3) \\
21(43.8)\end{array}$ & $\begin{array}{l}104(60.1) \\
69(39.9)\end{array}$ \\
\hline \multicolumn{4}{|l|}{$\begin{array}{l}\text { During the period of the } \\
\text { COVID-19 pandemic, how } \\
\text { frequently did you watch } \\
\text { television images of sick and dead } \\
\text { people caused by coronavirus? }\end{array}$} \\
\hline $\begin{array}{l}\text { Daily } \\
\text { Less than daily }\end{array}$ & $\begin{array}{l}58(46.0) \\
53(42.1)\end{array}$ & $\begin{array}{l}19(39.6) \\
21(43.8)\end{array}$ & $\begin{array}{l}77(44.3) \\
74(42.5)\end{array}$ \\
\hline $\begin{array}{l}\text { Respondents did not watch the TV } \\
\text { images of the devastation }\end{array}$ & $15(11.9)$ & $8(16.7)$ & $23(13.2)$ \\
\hline \multicolumn{4}{|l|}{$\begin{array}{l}\text { During the period of the } \\
\text { COVID-19 pandemic, how } \\
\text { frequently did you read } \\
\text { newspaper and internet articles } \\
\text { related to the pandemic? }\end{array}$} \\
\hline Daily & $72(57.1)$ & $29(60.4)$ & $101(58.0)$ \\
\hline Less than daily & $50(39.7)$ & $19(39.6)$ & $69(39.7)$ \\
\hline $\begin{array}{l}\text { Respondents did not read } \\
\text { newspaper and internet articles of } \\
\text { the devastation }\end{array}$ & $4(3.2)$ & $0(0.0)$ & $4(2.3)$ \\
\hline \multicolumn{4}{|l|}{$\begin{array}{l}\text { Did you lose your job due to the } \\
\text { COVID-19 pandemic? }\end{array}$} \\
\hline Yes & $14(11.1)$ & $7(14.6)$ & $21(12.1)$ \\
\hline No & $112(88.9)$ & $41(85.4)$ & $153(87.9)$ \\
\hline
\end{tabular}


Table 1. Cont.

\begin{tabular}{|c|c|c|c|}
\hline Variables & $\begin{array}{c}\text { in a } \\
\text { Relationship } \\
\text { n (\%) }\end{array}$ & $\begin{array}{c}\text { Not in a } \\
\text { Relationship } \\
\text { n (\%) }\end{array}$ & $\begin{array}{l}\text { Total } \\
\text { n (\%) }\end{array}$ \\
\hline \multicolumn{4}{|l|}{$\begin{array}{l}\text { Received sufficient support from } \\
\text { family and friends since the } \\
\text { COVID-19 pandemic declared }\end{array}$} \\
\hline Some-to-high level of support & $94(75.2)$ & $37(77.1)$ & $131(75.7)$ \\
\hline Limited or no support & $31(24.8)$ & $11(22.9)$ & $42(24.3)$ \\
\hline \multicolumn{4}{|l|}{$\begin{array}{l}\text { Received sufficient support from } \\
\text { Government of Canada since the } \\
\text { COVID-19 pandemic declared }\end{array}$} \\
\hline Some-to-high level of support & $38(30.9)$ & $11(23.9)$ & $49(29.0)$ \\
\hline Limited or no support & $85(69.1)$ & $35(76.1)$ & $120(71.0)$ \\
\hline \multicolumn{4}{|l|}{$\begin{array}{l}\text { Received sufficient support from } \\
\text { Government of Alberta since the } \\
\text { COVID-19 pandemic declared }\end{array}$} \\
\hline Some-to-high level of support & $32(29.9)$ & $9(20.0)$ & $41(27.0)$ \\
\hline Limited or no support & $75(70.1)$ & $36(80.0)$ & $111(73.0)$ \\
\hline \multicolumn{4}{|c|}{$\begin{array}{l}\text { Received sufficient support from } \\
\text { the employer since the COVID-19 } \\
\text { pandemic declared }\end{array}$} \\
\hline Some-to-high level of support & $90(72.6)$ & $34(70.8)$ & $124(72.1)$ \\
\hline Limited or no support & $34(27.4)$ & $14(29.2)$ & $48(27.9)$ \\
\hline \multicolumn{4}{|l|}{ Likely anxiety level } \\
\hline Low anxiety & $73(60.3)$ & $23(50.0)$ & $96(57.5)$ \\
\hline Moderate-to-severe anxiety & $48(39.7)$ & $23(50.0)$ & $71(42.5)$ \\
\hline
\end{tabular}

MH: Mental health.

Regarding COVID-19-related data, during the pandemic, 92\% (160) of respondents reported having been fearful about contracting the virus; $96.6 \%(168)$ were fearful about their close friends or family members contracting the coronavirus; $72.1 \%$ (124) reported having close friends or family members who have been sick from the coronavirus disease; $60.1 \%$ (104) had to self-isolate or self-quarantine due to COVID-19 symptoms, recent travel, or because of being in contact with someone who may have COVID-19; $44.3 \%$ (77) watched television images of sick and dead people caused by coronavirus, on a daily basis; $58 \%$ (101) read newspaper and internet articles related to the pandemic, on a daily basis; $12.1 \%$ (21) reported losing their job due to the COVID-19 pandemic; and received sufficient support from family and friends since the COVID-19 pandemic declared. Regarding receiving support since the COVID-19 pandemic was declared, respondents reported receiving a some-to-high level of support from: family and friends were $75.7 \%$ (131); from the Government of Canada were 29\% (49); from the Government of Alberta were 27\% (41); and from the employer were $72.1 \%$ (124). Moderate-to-severe anxiety was reported by $42.5 \%$ (71) of the respondents.

The univariate analysis in Table 2 included 30 demographic, clinical, and COVID-19related variables in relation to the likelihood of anxiety. Chi-squared or Fisher exact test revealed significant association between the likelihood of anxiety and 13 variables: employment status; history of depression diagnosis; history of anxiety diagnosis; history of any mental health diagnosis; history of antidepressant medications; history of Benzodiazepine medications; history of receiving any psychotropic medications; receiving mental health counselling; willingness to receiving mental health counselling; losing job due to COVID-19 pandemic; receiving sufficient support from family or friends; receiving sufficient support from Government of Canada; and receiving sufficient support from the employer, since the COVID-19 pandemic declared. No variables showed a near significant association with likely anxiety. 
Table 2. Chi-squared test of association between demographic, clinical, and COVID-19 characteristics and likely anxiety.

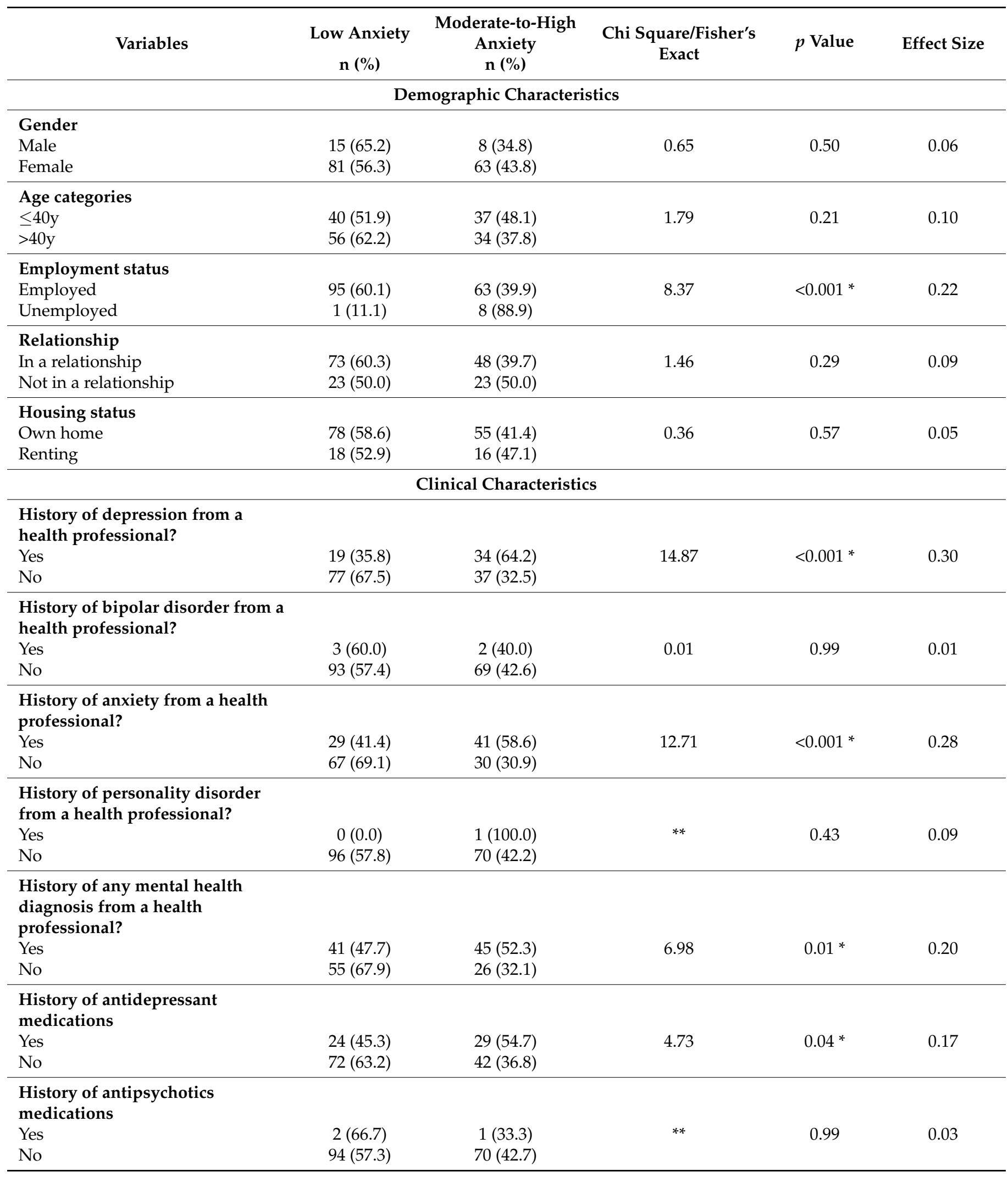


Table 2. Cont.

\begin{tabular}{|c|c|c|c|c|c|}
\hline Variables & $\begin{array}{c}\text { Low Anxiety } \\
\text { n (\%) }\end{array}$ & $\begin{array}{l}\text { Moderate-to-High } \\
\text { Anxiety } \\
\text { n (\%) }\end{array}$ & $\begin{array}{c}\text { Chi Square/Fisher's } \\
\text { Exact }\end{array}$ & $p$ Value & Effect Size \\
\hline \multicolumn{6}{|c|}{$\begin{array}{l}\text { History of benzodiazepines } \\
\text { medications }\end{array}$} \\
\hline Yes & $0(0.0)$ & $4(100.0)$ & & & \\
\hline No & $96(58.9)$ & $67(41.1)$ & $* *$ & $0.03 *$ & 0.18 \\
\hline \multicolumn{6}{|c|}{$\begin{array}{l}\text { History of mood stabilizers } \\
\text { medications }\end{array}$} \\
\hline Yes & $5(50.0)$ & $5(50.0)$ & & & \\
\hline No & $91(58.0)$ & $66(42.0)$ & 0.24 & 0.75 & 0.04 \\
\hline \multicolumn{6}{|c|}{$\begin{array}{l}\text { History of sleeping tablets } \\
\text { medications }\end{array}$} \\
\hline Yes & $8(44.4)$ & $10(55.6)$ & & & \\
\hline No & $88(59.1)$ & $61(40.9)$ & 1.40 & 0.31 & 0.09 \\
\hline \multicolumn{6}{|c|}{ On any psychotropic medication } \\
\hline Yes & $28(46.7)$ & $32(53.3)$ & 4.48 & 0.05 & 0.16 \\
\hline No & $68(63.6)$ & $39(36.4)$ & & & \\
\hline \multicolumn{6}{|c|}{$\begin{array}{l}\text { Respondents received MH } \\
\text { counselling in the past year }\end{array}$} \\
\hline Yes & $23(36.5)$ & $40(63.5)$ & 18.22 & $<0.001 *$ & 0.33 \\
\hline No & $73(70.2)$ & $31(29.8)$ & & & \\
\hline \multicolumn{6}{|c|}{$\begin{array}{l}\text { Respondents would like to } \\
\text { receive MH counselling }\end{array}$} \\
\hline Yes & $31(35.2)$ & $57(64.8)$ & 37.71 & $<0.001 *$ & 0.48 \\
\hline No & $65(82.3)$ & $14(17.7)$ & & & \\
\hline \multicolumn{6}{|c|}{ COVID-19-related Characteristics } \\
\hline \multicolumn{6}{|c|}{$\begin{array}{l}\text { Respondents who have been } \\
\text { fearful about contracting the } \\
\text { coronavirus during the pandemic }\end{array}$} \\
\hline Yes & $86(56.2)$ & $67(43.8)$ & 1.22 & 0.40 & 0.09 \\
\hline No & $10(71.4)$ & $4(28.6)$ & & & \\
\hline \multicolumn{6}{|c|}{$\begin{array}{l}\text { Respondents who have been } \\
\text { fearful about their close friends or } \\
\text { family members contracting the } \\
\text { coronavirus during the pandemic }\end{array}$} \\
\hline Yes & $90(55.9)$ & $71(44.1)$ & ** & 0.39 & 0.17 \\
\hline No & $6(100.0)$ & $0(0.0)$ & & & \\
\hline \multicolumn{6}{|c|}{$\begin{array}{l}\text { Respondents who reported } \\
\text { having close friends or family } \\
\text { members sick from the } \\
\text { coronavirus disease }\end{array}$} \\
\hline Yes & $69(58.5)$ & $49(41.5)$ & 0.38 & 0.60 & 0.05 \\
\hline No & $25(53.2)$ & $22(46.8)$ & & & \\
\hline \multicolumn{6}{|c|}{$\begin{array}{l}\text { Have you had to self-isolate or } \\
\text { self-quarantine due to COVID-19 } \\
\text { symptoms, recent travel, or } \\
\text { because you were in contact with } \\
\text { someone who may have } \\
\text { COVID-19? }\end{array}$} \\
\hline Yes & $58(56.9)$ & $44(43.1)$ & 0.01 & 0.99 & 0.01 \\
\hline No & $37(57.8)$ & $27(42.2)$ & & & \\
\hline
\end{tabular}


Table 2. Cont.

\begin{tabular}{|c|c|c|c|c|c|}
\hline Variables & $\begin{array}{c}\text { Low Anxiety } \\
\text { n (\%) }\end{array}$ & $\begin{array}{l}\text { Moderate-to-High } \\
\text { Anxiety } \\
\text { n (\%) }\end{array}$ & $\begin{array}{c}\text { Chi Square/Fisher's } \\
\text { Exact }\end{array}$ & $p$ Value & Effect Size \\
\hline \multicolumn{6}{|l|}{$\begin{array}{l}\text { During the period of the } \\
\text { COVID-19 pandemic, how } \\
\text { frequently did you watch } \\
\text { television images of sick and dead } \\
\text { people caused by coronavirus? }\end{array}$} \\
\hline Daily & $37(50.7)$ & $36(49.3)$ & 0.25 & 0.24 & 0.13 \\
\hline Less than daily & $47(64.4)$ & $26(35.6)$ & & & \\
\hline $\begin{array}{l}\text { Respondents did not watch the TV } \\
\text { images of the devastation }\end{array}$ & $12(57.1)$ & $9(42.9)$ & & & \\
\hline \multicolumn{6}{|l|}{$\begin{array}{l}\text { During the period of the } \\
\text { COVID-19 pandemic, how } \\
\text { frequently did you read } \\
\text { newspaper and internet articles } \\
\text { related to the pandemic? }\end{array}$} \\
\hline Daily & $51(52.6)$ & $46(47.4)$ & $* *$ & 0.31 & 0.12 \\
\hline Less than daily & $42(63.6)$ & $24(36.4)$ & & & \\
\hline $\begin{array}{l}\text { Respondents did not read } \\
\text { newspaper and internet articles of } \\
\text { the devastation }\end{array}$ & $3(75.0)$ & $1(25.0)$ & & & \\
\hline \multicolumn{6}{|l|}{$\begin{array}{l}\text { Did you lose your job due to the } \\
\text { COVID-19 pandemic? }\end{array}$} \\
\hline Yes & $5(25)$ & $15(75)$ & 9.81 & $<0.01 *$ & 0.24 \\
\hline No & $91(61.9)$ & $56(38.1)$ & & & \\
\hline \multicolumn{6}{|l|}{$\begin{array}{l}\text { Received sufficient support from } \\
\text { family and friends since the } \\
\text { COVID-19 pandemic declared }\end{array}$} \\
\hline Some-to-high level of support & $84(66.7)$ & $42(33.3)$ & 16.74 & $<0.001 *$ & 0.32 \\
\hline Limited or no support & $12(30.0)$ & $28(70.0)$ & & & \\
\hline \multicolumn{6}{|l|}{$\begin{array}{l}\text { Received sufficient support from } \\
\text { Government of Canada since the } \\
\text { COVID-19 pandemic declared }\end{array}$} \\
\hline Some-to-high level of support & $34(73.9)$ & $12(26.1)$ & 7.96 & $<0.01^{*}$ & 0.22 \\
\hline Limited or no support & $58(49.6)$ & $59(50.4)$ & & & \\
\hline \multicolumn{6}{|l|}{$\begin{array}{l}\text { Received sufficient support from } \\
\text { Government of Alberta since the } \\
\text { COVID-19 pandemic declared }\end{array}$} \\
\hline Some-to-high level of support & $27(71.1)$ & $11(28.9)$ & 2.37 & 0.18 & 0.13 \\
\hline Limited or no support & $62(56.9)$ & $47(43.1)$ & & & \\
\hline \multicolumn{6}{|l|}{$\begin{array}{l}\text { Received sufficient support from } \\
\text { the employer since the COVID-19 } \\
\text { pandemic declared }\end{array}$} \\
\hline Some-to-high level of support & $77(63.6)$ & $44(36.4)$ & 7.49 & 0.01 & 0.21 \\
\hline Limited or no support & $18(40.0)$ & $27(60.0)$ & & & \\
\hline
\end{tabular}

** Fisher's exact test. * $p<0.05$.

Table 3 illustrates the multivariate logistic regression model employed to predict likely anxiety among study respondents. The model included 10/13 out of the chi-squared predictor variables, after removal of two variables: history of any mental health diagnosis and history of antidepressant medications that showed high correlation with other variables $\left(\mathrm{r}_{\mathrm{s}}>0.7\right)$, and another variable: history of Benzodiazepine medications that showed no variability. 
Table 3. Logistic regression model for respondents' likelihood to present with moderate-tosevere anxiety.

\begin{tabular}{|c|c|c|c|c|c|c|c|c|}
\hline \multirow{2}{*}{ Characteristics } & \multirow{2}{*}{ B } & \multirow{2}{*}{ S.E. } & \multirow{2}{*}{ Wald } & \multirow{2}{*}{ df } & \multirow{2}{*}{$p$ Value } & \multirow{2}{*}{$\begin{array}{l}\text { Odd's } \\
\text { Ratio }\end{array}$} & \multicolumn{2}{|c|}{$95 \%$ C.I. for Odd's Ratio } \\
\hline & & & & & & & Lower & Upper \\
\hline Not employed & 2.046 & 1.327 & 2.375 & 1 & 0.123 & 7.734 & 0.574 & 104.303 \\
\hline $\begin{array}{l}\text { Having received depression } \\
\text { diagnosis from a health professional }\end{array}$ & 0.799 & 0.545 & 2.149 & 1 & 0.143 & 2.223 & 0.764 & 6.468 \\
\hline $\begin{array}{l}\text { Having received anxiety diagnosis } \\
\text { from a health professional }\end{array}$ & 0.870 & 0.561 & 2.411 & 1 & 0.121 & 2.388 & 0.796 & 7.166 \\
\hline $\begin{array}{l}\text { Have received mental health } \\
\text { counselling in the past year }\end{array}$ & 0.594 & 0.534 & 1.241 & 1 & 0.265 & 1.812 & 0.637 & 5.158 \\
\hline $\begin{array}{l}\text { Would like to receive mental health } \\
\text { counselling }\end{array}$ & 1.285 & 0.482 & 7.118 & 1 & $0.008 *$ & 3.616 & 1.406 & 9.296 \\
\hline $\begin{array}{l}\text { Not on any medication for mental } \\
\text { health concerns }\end{array}$ & -0.485 & 0.616 & 0.619 & 1 & 0.431 & 0.616 & 0.184 & 2.061 \\
\hline $\begin{array}{c}\text { Job loss due to the COVID-19 } \\
\text { pandemic (Yes) }\end{array}$ & 1.482 & 0.753 & 3.876 & 1 & $0.049 *$ & 4.401 & 1.007 & 19.241 \\
\hline $\begin{array}{c}\text { Received limited or no family } \\
\text { support since the COVID-19 } \\
\text { pandemic declared }\end{array}$ & 1.458 & 0.546 & 7.124 & 1 & $0.008 *$ & 4.296 & 1.473 & 12.530 \\
\hline $\begin{array}{l}\text { Received limited or no support from } \\
\text { Government of Canada since the } \\
\text { COVID-19 pandemic declared }\end{array}$ & 0.812 & 0.524 & 2.400 & 1 & 0.121 & 2.253 & 0.806 & 6.292 \\
\hline $\begin{array}{l}\text { Received limited or no support from } \\
\text { the employer since the COVID-19 } \\
\text { pandemic declared }\end{array}$ & -0.077 & 0.477 & 0.026 & 1 & 0.871 & 0.925 & 0.363 & 2.358 \\
\hline Constant & -2.922 & 0.584 & 24.993 & 1 & 0.000 & 0.054 & & \\
\hline
\end{tabular}

* Significance at $p<0.05$. C.I.: confidence interval. S.E.: standard error. Df: degree of freedom.

The model was statistically significant; $X^{2}(\mathrm{df}=12 ; \mathrm{n}=161)=75.39, p<0.001$, suggesting that the model could distinguish between respondents who did or did not exhibit likely anxiety during the COVID-19 pandemic. The model accounted for $37.4 \%$ (Cox and Snell $\mathrm{R}^{2}$ ) to $50.1 \%$ (Nagelkerke $\mathrm{R}^{2}$ ) of the variance. According to the goodness-of-fit statistic using the Hosmer-Lemeshow goodness-of-fit test, the model was adequately fit $\left(\mathrm{Chi}^{2}=10.0 ; p=0.27\right)$ and correctly classified $75.8 \%$ of cases

Only three variables, the desire to have MH support, receiving family support, and job loss due to the COVID-19 pandemic showed significant prediction of likely GAD in the model. Subscribers who reported that they would like to receive mental health support were more than three times likely to express moderate-to-severe anxiety during the COVID-19 pandemic, compared to those who were not willing to receive $\mathrm{MH}$ support $(\mathrm{OR}=3.39$; 95\% CI: 1.29-8.88), while controlling for the other variables. Likewise, respondents who reported losing their job during the pandemic were four times more likely to report likely anxiety, compared to those who did not lose their job (OR $=4.40$; 95\% CI: 1.01-19.24), after controlling for other model variables.

Similarly, the subscribers who reported receiving limited or no family support since the declaration of COVID-19 pandemic were almost five times more likely to report moderateto-severe anxiety, compared to those who received some-to-high level of family support (OR $=4.85$; 95\% CI: 1.56-15.03), while controlling for other variables. The largest contribution to the model was provided by the received family support variable (Wald = 7.46). 


\section{Discussion}

Our study examined the prevalence and the potential predictors of likely generalized anxiety disorder (GAD) development during the COVID-19 pandemic, among Fort McMurray's study participants. The prevalence of likely GAD was $42.5 \%$ in our study (based on self-reported GAD-7 scale). In comparison with other studies during similar disasters in the same community, the prevalence in our study was overall higher than what was previously reported 6 months and 18 months after the wildfires ( $20 \%$ and $18 \%$, respectively) $[19,30]$. This may reflect the stringent impact of the pandemic upon mental health and wellbeing. The time of sampling may be an additional factor; where the current survey was carried out during the continuous pandemic when there was still so much uncertainty, while the wildfire surveys were conducted at 6 and 18 months after the wildfires. On the other hand, this prevalence is consistent with other studies of the same variable during the pandemic in different places such as China and other parts of Canada [31,32] in which GAD prevalence was reported at $44.6 \%$ and $46.7 \%$, respectively. A lower prevalence of anxiety was reported in a systematic review conducted during the pandemic (27.3-39.6\%); however, the study reported a marked discrepancy in such values between females and males at $48 \%$ and $28 \%$, respectively [5]. Given that the majority of our respondents were females, the reported high anxiety levels may be related to this particular category. Consequently, it appears that, contrary to our stated hypothesis, the prevalence of moderate-to-high anxiety in FMM, a community with a history of two previous natural disasters, was aligned with and in some consideration, even less than (when considering the female majority of the sample) than the prevalence of moderate-to-high anxiety experienced in other communities during the COVID-19 pandemic. This may be attributed to either the unprecedented effect of the pandemic that triggered the maximum levels of mental distress regardless of the previous traumas, or to some extent, to the adaptability and resilience developed by FMM residents after experiencing frequent traumas.

Among sociodemographic characteristics, employment status showed a significant association with the likelihood of anxiety, where the majority of unemployed people reported moderate-to-high anxiety symptoms; however, the limited number of respondents in this category $(n=9)$ may impair any meaningful interpretation of such a finding. However, given the fact that the ability to work from home during lockdown is a consideration specific to pandemic trauma (compared to the wildfire or flooding those residents had also experienced), it stands to reason that unemployment would be associated with significant increases in anxiety. Other than employment status, none of the demographic characteristics showed an association with anxiety symptoms during the pandemic. Although females and the younger age expressed a higher prevalence of anxiety symptoms, compared to males and older age groups, respectively, the difference was minimal and not significant. This contradicts other studies, where the younger age groups were found to be more prone to express mental health stresses, including anxiety symptoms during the pandemic, compared to the older age groups $[5,12]$. The relatively small sample size of our study may not have elicited that difference as reported in other studies.

The three variables that correlated with high odds of likely GAD in our study were the need to receive mental health support, job loss due to the pandemic, and the limited/lack of family support since the declaration of the pandemic. Respondents who reported their need to receive or were receiving mental health support were consistently reported, in other studies, to be at a higher risk of expressing mental health symptoms [14,30]. However, this finding may need to be interpreted cautiously and in the context of the association relationship between the need of support and the underlying pathology. Although this need may be perceived as a serious indicator for a possible presence or development of psychopathology, the reverse may be the case, where the underlying anxiety symptoms may have triggered the excessive $\mathrm{MH}$ need, particularly during the pandemic

In the same context, our results did not find underlying mental health conditions to significantly predict the presence of likely anxiety. Although those who reported having a diagnosis of depression or anxiety at the time of the survey were from two to three folds 
more likely to express anxiety symptoms, these associations were not statistically significant. This contrasts with the previous literature, in which people who had prior mental health conditions were at a high risk of developing mental illness post-disaster [14,33,34]. This may be explained by those who have a well-diagnosed mental health condition may be in a better position than those who are yet to be diagnosed, as receiving regular therapeutic support may, otherwise guard against further symptomatology. However, the timing of data collection in relation to the pandemic phase may also have a role. In one study, for example, the authors found that the pre-existing mental health condition was a significant predictor for psychological distress early in the study phase, while the effect disappeared later in the study [13]. Similarly, they found that people with increasing mental health symptoms have experienced resilience early in the pandemic, which faded later, and in contrast to those who reported high scores in the early weeks, they showed considerable improvement with the pandemic progression [13].

Our results showed that losing a job due to the pandemic represented a significant risk factor of developing anxiety symptoms, compared to those who did not lose their jobs in our study cohort. This finding is consistent with previous research which reported losing a job or job insecurity was linked to various mental health adversities including depression and psychological distress $[35,36]$. People who lost their job in an Australian cohort were four times more likely to express severe psychological distress compared to the employed people during the pandemic [36]. Furthermore, people who kept working during the pandemic demonstrated better mental and physical health, and life satisfaction, compared to the people who stopped working; these results were fairly similar for those who worked at their home or at their office [37].

In our study, the role of family or friends' support during the COVID-19 pandemic was a determinant for the likelihood of GAD. Those who reported receiving limited or no support from the family or friends were more prone to likely GAD, compared to those receiving some-to-high level of support. Several studies supported such a finding, exploring the role of the family and the social support in ameliorating anxiety and improving coping and resilience, among other distresses during and after disasters and pandemics $[14,19,36,38]$. Moreover, having sufficient support prior to a disaster may act as a barrier against psychological trauma developing usually after the crisis [9]. Additionally, having poor relationships or lack of social support, prior to a disaster is accompanied by more psychopathologies [33].

\section{Limitations of the Study}

Our study has several limitations. Firstly, the sample size is relatively small; thus, our results may need to be replicated with other large-scale studies. Secondly, the majority of our respondents were females, which may limit the generalizability of the data. Thirdly, our study results were limited by the frequency of the reported conditions; for example, none of the study participants reported a history of psychotic disorder, on which we could not establish the relationship between the history of psychotic disorder and the present condition of anxiety symptoms. Fourth, given that the survey was shared via e-mail by community partners, it was not possible to specify how many individuals received the survey links. The study response rate was calculated using the number of unique individuals who clicked on the link as the denominator, rather than the number who received the link, as the latter could not be determined, which could lead to an overestimation of the response rate. Finally, the measurement scale for likely GAD was self-reported and was not supported by objective and detailed clinical assessment.

\section{Conclusions}

This study highlighted the mental health burdens-mainly anxiety-during the COVID-19 pandemic in FMM. It adds to the clinical and research evidence related to the field of trauma and disasters. The study identified the prevalence and the correlates of the likelihood of developing GAD among residents of Fort McMurray, Canada, during 
the COVID-19 pandemic. Against our expectations, although we found a high level of anxiety experienced among FMM residents during the current pandemic when compared to other previous disasters, this level was fairly aligned with the results of other reports within non-traumatized communities during the COVID-19 pandemic. On the other hand, in line with our hypothesis, the received support from the family and friends and the reported need for mental health support are substantially related to likely GAD, although none of the demographic or underlying clinical conditions could predict likely anxiety. Such results may need cautious interpretation in light of the small sample size and limited reportedly history of clinical conditions. Therefore, it is imperative for the research and policy to mobilize the resources that widely examine and safeguard against developing anxiety symptoms, with a clear understanding of the leading causes that contribute to such outcomes during pandemics, in order to improve personal functionality and achieve a better quality of life.

\section{Key Points}

1. The prevalence of anxiety symptoms among Fort McMurray residents was $42.5 \%$ based on GAD-7, a self-reported measure;

2. The subscribers who stated that they would like to receive mental health support were three times more likely to demonstrate likely anxiety;

3. The lack of family/friend support during the COVID-19 pandemic demonstrated a significant risk factor for developing anxiety symptoms;

4. Similarly, losing the job during the COVID-19 pandemic was significantly related to the development of anxiety symptoms.

Author Contributions: The study was conceived and designed by V.I.O.A.; R.S. conducted the data analysis and drafted the initial manuscript. E.E. contributed to data collection. All authors contributed to study design, reviewing, and revising the initial draft manuscript: R.S., E.E., N.N., B.A., H.P., G.O.-D., M.K.A., W.M., E.O., F.O. and V.I.O.A. All authors have read and agreed to the published version of the manuscript.

Funding: This study was supported by grants from the Mental Health Foundation and the Douglas Harden Trust Fund.

Institutional Review Board Statement: The study was conducted in accordance with the Declaration of Helsinki, Study approval was granted by Alberta Health Research Ethics Committee (Pro00066054).

Informed Consent Statement: Informed consent was implied from all subjects involved in the study, by completing the survey.

Data Availability Statement: Data is available upon reasonable request to the submitting author.

Acknowledgments: Support with survey link distribution was received from the Fort McMurray Public and Catholic School Boards, Keyano College, and the Canadian Mental Health Association.

Conflicts of Interest: The authors declare no conflict of interest.

\section{References}

1. Hassannia, L.; Taghizadeh, F.; Moosazadeh, M.; Zarghami, M.; Taghizadeh, H.; Dooki, A.F.; Fathi, M.; Alizadeh-Navaei, R.; Hedayatizadeh-Omran, A.; Dehghan, N. Anxiety and Depression in Health Workers and General Population During COVID-19 in IRAN: A Cross-Sectional Study. Neuropsychopharmacol. Rep. 2021, 41, 40-49. [CrossRef] [PubMed]

2. da Silva, F.C.T.; Neto, M.L.R. Psychological effects caused by the COVID-19 pandemic in health professionals: A systematic review with meta-analysis. Prog. Neuropsychopharmacol. Biol. Psychiatry 2021, 104, 110062. [CrossRef]

3. Zhang, X.; Shi, X.; Wang, Y.; Jing, H.; Zhai, Q.; Li, K.; Zhao, D.; Zhong, S.; Song, Y.; Zhang, F.; et al. Risk Factors of Psychological Responses of Chinese University Students During the COVID-19 Outbreak: Cross-sectional Web-Based Survey Study. J. Med. Internet Res. 2021, 23, e29312. [CrossRef]

4. Zhou, S.-J.; Zhang, L.-G.; Wang, L.-L.; Guo, Z.-C.; Wang, J.-Q.; Chen, J.-C.; Liu, M.; Chen, X.; Chen, J.-X. Prevalence and socio-demographic correlates of psychological health problems in Chinese adolescents during the outbreak of COVID-19. Eur. Child Adolesc. Psychiatry 2020, 29, 749-758. [CrossRef] 
5. $\quad$ Pashazadeh Kan, F.; Raoofi, S.; Rafiei, S.; Khani, S.; Hosseinifard, H.; Tajik, F.; Raoofi, N.; Ahmadi, S.; Aghalou, S.; Torabi, F.; et al. A systematic review of the prevalence of anxiety among the general population during the COVID-19 pandemic. J. Affect. Disord. 2021, 293, 391-398. [CrossRef]

6. Salari, N.; Hosseinian-Far, A.; Jalali, R.; Vaisi-Raygani, A.; Rasoulpoor, S.; Mohammadi, M.; Rasoulpoor, S.; Khaledi-Paveh, B Prevalence of stress, anxiety, depression among the general population during the COVID-19 pandemic: A systematic review and meta-analysis. Glob. Health 2020, 16, 57. [CrossRef]

7. Deng, J.; Zhou, F.; Hou, W.; Silver, Z.; Wong, C.Y.; Chang, O.; Huang, E.; Zuo, Q.K. The prevalence of depression, anxiety, and sleep disturbances in COVID-19 patients: A meta-analysis. Ann. N. Y. Acad. Sci. 2021, 1486, 90-111. [CrossRef] [PubMed]

8. SAMHSA. Disaster Technical Assistance Center. Supplemental Research Bulletin. Disasters and People With Serious Mental Illness. 2019. Available online: https:/ / www.samhsa.gov/sites/default/files/disasters-people-with-serious-mental-illness.pdf (accessed on 21 September 2021).

9. Mao, W.; Agyapong, V.I.O. The Role of Social Determinants in Mental Health and Resilience after Disasters: Implications for Public Health Policy and Practice. Front. Public Health 2021, 9, 658528. [CrossRef]

10. Mental Health and Psychosocial Considerations during the COVID-19 Outbreak. 18 March 2020. Available online: https://apps who.int/iris/bitstream/handle/10665/331490/WHO-2019-nCoV\%20-MentalHealth-2020.1-eng.pdf?sequence=1 (accessed on 22 August 2021).

11. Gao, J.; Zheng, P.; Jia, Y.; Chen, H.; Mao, Y.; Chen, S.; Wang, Y.; Fu, H.; Dai, J. Mental health problems and social media exposure during COVID-19 outbreak. PLOS ONE 2020, 15, e0231924.

12. Nwachukwu, I.; Nkire, N.; Shalaby, R.; Hrabok, M.; Vuong, W.; Gusnowski, A.; Surood, S.; Urichuk, L.; Greenshaw, A.J.; Agyapong, V.I.O. COVID-19 Pandemic: Age-Related Differences in Measures of Stress, Anxiety and Depression in Canada. Int. J. Environ. Res. Public Health 2020, 17, 6366. [CrossRef]

13. McPherson, K.E.; McAloney-Kocaman, K.; McGlinchey, E.; Faeth, P.; Armour, C. Longitudinal analysis of the UK COVID-19 Psychological Wellbeing Study: Trajectories of anxiety, depression and COVID-19-related stress symptomology. Psychiatry Res. 2021, 304, 114138. [CrossRef] [PubMed]

14. Shalaby, R.; Adu, M.K.; Andreychuk, T.; Eboreime, E.; Gusnowski, A.; Vuong, W.; Surood, S.; Greenshaw, A.J.; Agyapong, V.I.O. Prevalence, Demographic, and Clinical Correlates of Likely PTSD in Subscribers of Text4Hope during the COVID-19 Pandemic. Int. J. Environ. Res. Public Health 2021, 18, 6227. [CrossRef] [PubMed]

15. Kunzler, A.M.; Rothke, N.; Gunthner, L.; Stoffers-Winterling, J.; Tuscher, O.; Coenen, M.; Rehfuess, E.; Schwarzer, G.; Binder, H.; Schmucker, C.; et al. Mental burden and its risk and protective factors during the early phase of the SARS-CoV-2 pandemic: Systematic review and meta-analyses. Glob. Health 2021, 17, 34. [CrossRef] [PubMed]

16. Malakoutikhah, A.; Zakeri, M.A.; Salehi Derakhtanjani, A.; Dehghan, M. Anxiety, anger, and mindfulness as predictors of general health in the general population during COVID-19 outbreak: A survey in southeast Iran. J. Community Psychol. 2021, 17, 34. [CrossRef]

17. Holmes, E.A.; O'Connor, R.C.; Perry, V.H.; Tracey, I.; Wessely, S.; Arseneault, L.; Ballard, C.; Christensen, H.; Cohen Silver, R.; Everall, I.; et al. Multidisciplinary research priorities for the COVID-19 pandemic: A call for action for mental health science. Lancet Psychiatry 2020, 7, 547-560. [CrossRef]

18. Brown, M.R.G.; Agyapong, V.; Greenshaw, A.J.; Cribben, I.; Brett-MacLean, P.; Drolet, J.; McDonald-Harker, C.; Omeje, J.; Mankowsi, M.; Noble, S.; et al. Significant PTSD and Other Mental Health Effects Present 18 Months after the Fort Mcmurray Wildfire: Findings from 3070 Grades 7-12 Students. Front. Psychiatry 2019, 10, 623. [CrossRef]

19. Agyapong, V.I.O.; Hrabok, M.; Juhas, M.; Omeje, J.; Denga, E.; Nwaka, B.; Akinjise, I.; Corbett, S.E.; Moosavi, S.; Brown, M.; et al Prevalence Rates and Predictors of Generalized Anxiety Disorder Symptoms in Residents of Fort McMurray Six Months after a Wildfire. Front. Psychiatry 2018, 9, 345. [CrossRef]

20. Agyapong, V.I.O.; Juhas, M.; Omege, J.; Denga, E.; Nwaka, B.; Akinjise, I.; Corbett, S.E.; Brown, M.; Chue, P.; Li, X.-M.; et al. Prevalence Rates and Correlates of Likely Post-Traumatic Stress Disorder in Residents of Fort McMurray 6 Months after a Wildfire. Int. J. Ment. Health Addict. 2021, 19, 632-650. [CrossRef]

21. Agyapong, V.I.O.; Juhás, M.; Brown, M.R.G.; Omege, J.; Denga, E.; Nwaka, B.; Akinjise, I.; Corbett, S.E.; Hrabok, M.; Li, X.-M.; et al. Prevalence Rates and Correlates of Probable Major Depressive Disorder in Residents of Fort McMurray 6 Months after a Wildfire. Int. J. Ment. Health Addict. 2019, 17, 120-136. [CrossRef]

22. Brown, M.R.G.; Agyapong, V.; Greenshaw, A.J.; Cribben, I.; Brett-MacLean, P.; Drolet, J.; McDonald-Harker, C.; Omeje, J.; Mankowsi, M.; Noble, S.; et al. After the Fort McMurray wildfire there are significant increases in mental health symptoms in grade 7-12 students compared to controls. BMC Psychiatry 2019, 19, 18.

23. Insurance Bureau of Canada; CISION. Fort McMurray Flooding Causes over \$228 Million in Insured Damage. 1 June 2020. Available online: https: / / www.newswire.ca/news-releases / fort-mcmurray-flooding-causes-over-228-million-in-insured-damage837624948.html (accessed on 22 August 2021).

24. Pruden, J.G. COVID-19 Hot Spot Fort McMurray Weathers Its Latest Disaster with Frustration and Resolve. The Globe and Mail. Available online: https:/ / www.theglobeandmail.com/canada/alberta/article-covid-19-hot-spot-fort-mcmurray-weathers-itslatest-disaster-with/ (accessed on 3 October 2021).

25. Municipal Census Peport. Census 2018. Available online: https://www.rmwb.ca/en/permits-and-development/resources/ Documents/Latest-Census-Report-2018.pdf (accessed on 11 October 2021). 
26. Harris, P.A.; Taylor, R.; Thielke, R.; Payne, J.; Gonzalez, N.; Conde, J.G. Research electronic data capture (REDCap)—A metadatadriven methodology and workflow process for providing translational research informatics support. J. Biomed. Inf. 2009, 42, 377-381. [CrossRef]

27. Johnson, S.; Ulvenes, P.; Oktedalen, T.; Hoffart, A. Psychometric Properties of the General Anxiety Disorder 7-Item (GAD-7) Scale in a Heterogeneous Psychiatric Sample. Front. Psychol. 2019, 10, 1713. [CrossRef] [PubMed]

28. Spitzer, R.L.; Kroenke, K.; Williams, J.B.; Lowe, B. A brief measure for assessing generalized anxiety disorder: The GAD-7. Arch. Intern. Med. 2006, 166, 1092-1097. [CrossRef]

29. IBM. IBM Support. Release Notes-IBM®SPSS®Statistics 25.0. Available online: https://www.ibm.com/support/pages/releasenotes-ibm\%C2\%AE-spss\%C2\%AE-statistics-250 (accessed on 8 December 2020).

30. Moosavi, S.; Nwaka, B.; Akinjise, I.; Corbett, S.E.; Chue, P.; Greenshaw, A.J.; Silverstone, P.H.; Li, X.-M.; Agyapong, V.I.O. Mental Health Effects in Primary Care Patients 18 Months after a Major Wildfire in Fort McMurray: Risk Increased by Social Demographic Issues, Clinical Antecedents, and Degree of Fire Exposure. Front. Psychiatry 2019, 10, 683. [CrossRef] [PubMed]

31. Lai, J.; Ma, S.; Wang, Y.; Cai, Z.; Hu, J.; Wei, N.; Wu, J.; Du, H.; Chen, T.; Li, R.; et al. Factors Associated With Mental Health Outcomes Among Health Care Workers Exposed to Coronavirus Disease 2019. JAMA Netw. Open 2020, 3, e203976. [CrossRef]

32. Hrabok, M.; Nwachukwu, I.; Gusnowski, A.; Shalaby, R.; Vuong, W.; Surood, S.; Li, D.; Greenshaw, A.J.; Agyapong, V.I.O. Mental Health Outreach via Supportive Text Messages during the COVID-19 Pandemic: One-week Prevalence and Correlates of Anxiety Symptoms. Can. J. Psychiatry 2021, 66, 59-61. [CrossRef] [PubMed]

33. Goldmann, E.; Galea, S. Mental health consequences of disasters. Annu. Rev. Public Health 2014, 35, 169-183. [CrossRef] [PubMed]

34. Maguen, S.; Neria, Y.; Conoscenti, L.; Litz, B. Depression and Prolonged Grief in the Wake of Disasters. In Mental Health and Disasters; Cambridge University Press: Cambridge, UK, 2009; pp. 116-130.

35. Burgard, S.A.; Kalousova, L.; Seefeldt, K.S. Perceived job insecurity and health: The Michigan Recession and Recovery Study. J. Occup. Environ. Med. 2012, 54, 1101-1106. [CrossRef]

36. Collie, A.; Sheehan, L.; Vreden, C.V.; Grant, G.; Whiteford, P.; Petrie, D.; Sim, M.R. Psychological Distress Among People Losing Work During the COVID-19 Pandemic in Australia. medRxiv 2020. [CrossRef]

37. Zhang, S.X.; Wang, Y.; Rauch, A.; Wei, F. Unprecedented disruption of lives and work: Health, distress and life satisfaction of working adults in China one month into the COVID-19 outbreak. Psychiatry Res. 2020, 288, 112958. [CrossRef]

38. Carr, V.J.; Lewin, T.J.; Webster, R.A.; Hazell, P.L.; Kenardy, J.A.; Carter, G.L. Psychosocial sequelae of the 1989 Newcastle earthquake: I. Community disaster experiences and psychological morbidity 6 months post-disaster. Psychol. Med. 1995, 25, 539-555. [CrossRef] [PubMed] 